\title{
Neuronal nitric oxide synthase, as a downstream signaling molecule of c-jun, regulates the survival of differentiated PC12 cells
}

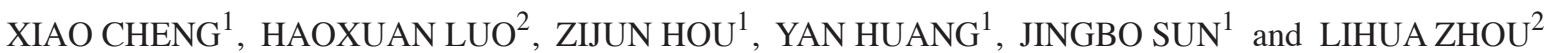 \\ ${ }^{1}$ Department of Encephalopathy, Encephalopathy Center, Guangdong Provincial Hospital of Traditional Chinese Medicine, \\ Guangzhou, Guangdong 510120; ${ }^{2}$ Department of Anatomy, Zhongshan School of Medicine, \\ Sun Yat-Sen University, Guangzhou, Guangdong 510080, P.R. China
}

Received November 25, 2013; Accepted May 9, 2014

DOI: $10.3892 / \mathrm{mmr} .2014 .2415$

\begin{abstract}
The high expression of c-jun and neuronal nitric oxide synthase (nNOS) generally occurs in neurons following the generation of various animal models of central neuronal diseases. However, the mechanism between them in neuronal disease remains to be elucidated. Our previous studies demonstrated that the expression of c-jun always occurs prior to expression of nNOS in motoneuron injuries and suppression of c-jun expression by c-jun siRNA decreased $n$ NOS expression in differentiated PC12 cells. The present study aimed to examine whether there was an association of up and downstream regulation or crosstalk between c-jun and nNOS in neurons. Using a culture of differentiated PC12 cells in vitro, the expression of nNOS and c-jun in cells was investigated by immunofluorescence. The nNOS inhibitor 7-nitroindazole (7-NI) was used in differentiated PC12 cells to downregulate the expression of nNOS. The optimal concentration of 7-NI on the viability and survival of cultured differentiated $\mathrm{PC} 12$ cells was selected using a 3-(4,5-dimethylthiazol-2-yl)-2,5-diphenyltetrazolium assay and the effects of 7-NI on the activity of constitutive nitric oxide synthase (cNOS) in differentiated PC12 cells were determined using a NOS Activity Detection kit. The effects of 7-NI on the gene expression of nNOS and c-jun were detected by western blot analysis. The results from the immunofluorescence demonstrated that the c-jun and nNOS protein were constantly expressed in PC12 cells. The cell viability of
\end{abstract}

Correspondence to: Professor Jingbo Sun, Department of Encephalopathy, Encephalopathy Center, Guangdong Provincial Hospital of Traditional Chinese Medicine, no. 111 Dade Road, Guangzhou, Guangdong 510120, P.R. China

E-mail: sunhuachen96@163.com

Professor Lihua Zhou, Department of Anatomy, Zhongshan School of Medicine, Sun Yat-Sen University, no. 74 Zhongshan Er Road, Guangzhou, Guangdong 510080, P.R. China

E-mail: zhoulih@mail.sysu.edu.cn

Key words: neuronal nitric oxide synthase, c-jun, PC12 cells differentiated PC12 cells were significantly inhibited by treatment with 200 and $400 \mu \mathrm{mol} / 1$ 7-NI, and the expression levels of the nNOS protein were significantly inhibited by treatment with $200 \mu \mathrm{mol} / 1$ 7-NI. However, 7-NI had no significant effect on the protein expression level of c-jun and the total activities of cNOS. Based on our previous studies, which revealed that the nNOS gene was a downstream signaling molecule of the JNK/c-jun signaling pathway in cultured neurons, the expression of nNOS downstream was able to be regulated by c-jun which was the upstream molecule. Therefore, these results indicated that the association between them involved up and downregulation instead of crosstalk.

\section{Introduction}

Currently, the therapies for nerve injury focus on revealing the molecular mechanism of injured neuron death and regeneration. It was reported that nerve injuries caused by several factors could induce molecular apoptosis and regenerative events in neurons, and functional recovery of the nervous system depended on the interaction between apoptosis and regeneration signals (1). Therefore, the objective of studies switched to enhancing the activities of regenerative signaling pathways and inhibiting the transduction of apoptotic signals (2). Nitric oxide synthase (NOS), which is able to generate nitric oxide (NO) by utilizing L-arginine as a substrate and nicotinamide adenine dinucleotide phosphate (NADPH) as the hydrogen delivery body, can be divided into neuronal nitric oxide synthase (nNOS), endothelial nitric oxide synthase (eNOS) and inducible nitric oxide synthase (iNOS). According to their tissue sources, nNOS and eNOS are collectively referred to as constitutive nitric oxide synthase (cNOS) (3). The majority of nNOS is located in the neurons, only some of which is in the nerve fibers surrounded by cerebrovascular fluid. In addition, it is generally expressed at a low level and as a catalyst to generate a small quantity of NO. Therefore, it is important in the control of cognitive function, synaptic plasticity and the neurosecretory system (4). However, nNOS is a 'double-edged sword' during injuries of the nervous system, in other words, it has been demonstrated to have positive and negative roles during neural regeneration 
in different disease models (5). It is well documented that nNOS is a factor which is able to promote the survival of differentiated PC12 cells (6). In addition, several studies have revealed that transcription factors also have critical roles in the death and regeneration procedures of injury-induced neurons in the peripheral and central nervous system (7). There were types of inducible transcriptional factors closely associated with the injury of neuron axons in the central nervous system, however, only the proto-oncogene-coding c-jun protein and its family member JunD were constantly expressed following axon injuries (8). The c-jun N-terminal kinase (JNK), also termed the stress activated protein kinase, was one of the downstream signaling molecules in the mitogen-activated protein kinase pathway, which was activated via cytokines and growth factors following stimulation by ultraviolet, ionizing radiation and heat shock $(9,10)$. In addition, c-jun was the main transcriptional factor expressed by cerebral neurons during degenerative procedures, including ischemic tolerance and dementia (11). Furthermore, as a member of the AP-1 family, c-jun may be a controller of target genes by combining with certain other transcription factors, including Jun, Fos and ATF (12). Similarly to nNOS, c-jun was demonstrated to have a dual role in injuries to the nervous system (13). According to previous studies, the activation of c-jun mediated the apoptotic process in differentiated PC12 cells and other neurons $(14,15)$.

The expression level of c-jun has been demonstrated to be upregulated when the expression level of nNOS is inhibited and the survival rate of neurons enhanced by utilizing neurotrophic factors or antioxidants, indicating a close association between c-jun and nNOS (1,16,17). Furthermore, the differentiation of PC12 cells induced the upregulation of c-jun and nNOS expression in a study by Cheng et al (18). The activation of c-jun mediated the apoptosis of PC12 cells and the nNOS protein maintained the survival of PC12 cells $(6,14)$. Finally, the application of c-jun siRNA for downregulating the expression of c-jun was previously indicated to reduce the expression level of nNOS in differentiated PC12 cells $(18,19)$. Therefore, in order to clarify whether the association between c-jun and nNOS involved up and downregulation or crosstalk, pheochromocytoma differentiated PC12 cells were selected as the cell model and 7-nitroindazole (7-NI) as the inhibitor of nNOS to downregulate the expression level of nNOS. Thus, the effects of the changes in the nNOS gene on the expression level of c-jun and the association between c-jun and nNOS in neurons were determined in order to improve our understanding of the molecular mechanisms underlying neural diseases.

\section{Materials and methods}

Cell culture. Differentiated PC12 cells, a rat neuronal cell line derived from pheochromocytoma cells, were purchased from the Cell Resource Center, Shanghai Institute for Biological Sciences, China Scientific Academy (Shanghai, China). The culture of differentiated PC12 cells was established by modification of a previously described procedure $(18,19)$. Cells were maintained in Dulbecco's modified Eagle's medium (DMEM, high glucose; Gibco-BRL, Carlsbad, CA, USA) supplemented with $10 \%$ fetal bovine serum and antibiotics $(100 \mathrm{U} / \mathrm{ml}$ penicillin A and $100 \mathrm{~g} / 1$ streptomycin; Gibco-BRL) at $37^{\circ} \mathrm{C}$ in a humidified $5 \% \mathrm{CO}_{2}$ incubator. The monolayer cells were subcultured once they reached $\sim 80 \%$ confluence as previously described (19). In brief, cells were washed 2-3 times with D-Hanks' solution following aspirating the culture medium, and then $0.25 \%$ trypsin was added for digestion at $37^{\circ} \mathrm{C}$. Digestion was not terminated by appropriate high glucose DMEM complete medium until the cells became rounded, the cell processes retracted and the cell gap became large. Monolayer cells were collected by centrifugation for $3 \mathrm{~min}$ at $90 \mathrm{x} \mathrm{g}$ and diluted and plated on new flasks for subculture. Subsequent experiments were performed following 3-4 passages when cells were in a stable state.

The other three sets of PC12 cells were treated with growth medium only (normal control), dimethyl sulfoxide (DMSO; solvent control group) and 7-NI (nNOS inhibitor group; Cell Signaling Technology, Danvers, MA, USA), respectively.

Immunofluorescence staining for $n N O S$ and c-jun expression in vitro. Differentiated PC12 cells were seeded onto poly-lysine-coated 96-well plates at a density of $2 \times 10^{4}$ cells $/ \mathrm{ml}$ for adherence overnight. Subsequently, the cultured cells were fixed with $4 \%$ paraformaldehyde in phosphate-buffered saline (PBS) at room temperature (RT) for $15 \mathrm{~min}$. Following rinsing three times with PBS, the cells were treated with $0.3 \%$ Triton $\mathrm{X}-100$ and $0.1 \%$ bovine serum albumin (BSA) in PBS at RT for $30 \mathrm{~min}$, and incubated with either rabbit anti-nNOS primary antibody (1:2,000; Cell Signaling Technology), rabbit anti-c-jun primary antibody (1:400; Cell Signaling Technology) or serum at $4^{\circ} \mathrm{C}$ overnight. Then, following washing three times with PBS, cells were incubated with fluorescein isothiocyanate-conjugated anti-rat IgG (1:200; Cell Signaling Technology) or rhodamine-conjugated anti-rabbit IgG (1:800; Cell Signaling Technology) at RT for an additional $2 \mathrm{~h}$ in the dark, respectively. Following rinsing the cells a further three times with PBS, the nuclei were stained with Hoechst 33258 for 20 min prior to being washed briefly with PBS and then visualized under a Leica DMI4000 B fluorescence microscope (Leica Microsystems GmbH, Wetzlar, Germany). Cells stained without primary or secondary antibodies served as negative controls.

\section{3-(4,5-dimethylthiazol-2-yl)-2,5-diphenyltetrazolium (MTT)} assay. The viability of differentiated PC12 cells was assessed by measuring the dehydrogenase activity in the culture, which was indicated by MTT tests (mitochondrial activity, as a measure of cell death) as described previously (20). Briefly, following incubation with DMSO as a solvent control or 10, 50, 100, 200 and $400 \mu \mathrm{mol} / 1$ 7-NI in 96-well microtiter plates for $24 \mathrm{~h}$, the medium was removed and the cells were incubated in $150 \mu \mathrm{l}$ culture medium per well. MTT $(10 \mu 1,0.5 \mathrm{mg} / \mathrm{ml}$ per well) was added to the culture and incubated for an additional $4 \mathrm{~h}$ at $37^{\circ} \mathrm{C}$ and $5 \% \mathrm{CO}_{2}$. DMSO $(150 \mu \mathrm{l})$ was added to each well to solubilize the formazan salt following removal of the media. Finally, the resultant purple azo-dye was detected at $570 \mathrm{~nm}$ with a SpectraMax M5 plate reader (Molecular Devices, Sunnyvale, CA, USA). Untreated PC12 cells were used as a control with $100 \%$ viability. The cell viability in treated PC12 cells was calculated as a percentage relative to the untreated PC12 cells.

Determination of cNOS activity. Differentiated PC12 cells were plated onto six-well plates at a density of $1 \times 10^{6}$ cells $/ \mathrm{ml}$ 
( $2 \mathrm{ml} /$ well). The total protein was extracted from the cells of each group $24 \mathrm{~h}$ after different interventions. iNOS activity and total NOS activity in the cells were measured using a NOS Activity Detection kit (Nanjing Jiancheng Bioengineering Institute, Nanjing, China), which assessed NOS activity by measuring the conversion of L-[14C]-arginine to L-[14C]-citrulline $(21,22)$. The total NOS activity was determined by incubating samples $(50 \mu \mathrm{l})$ for $15 \mathrm{~min}$ at $37^{\circ} \mathrm{C}$ in a reaction mixture containing buffer

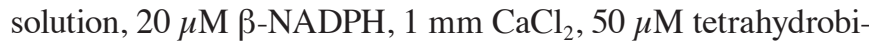
opterin (BH4) and $1 \mu \mathrm{Ci} / \mathrm{ml} \mathrm{L}$-[14C]-arginine. iNOS activity was measured by omitting calcium and adding $1 \mathrm{~mm}$ ethylenediaminetetraacetic acid (EDTA) to the reaction mixture $(50 \mu \mathrm{l})$ for $60 \mathrm{~min}$ at $37^{\circ} \mathrm{C}$. The reaction was terminated by the addition of $1 \mathrm{ml}$ ice-chilled buffer containing $30 \mathrm{~mm}$ HEPES and $3 \mathrm{~mm}$ EDTA ( $\mathrm{pH} 5.5$ ), following which the reaction mix was applied to Dowex 50WX8 columns (Sigma-Aldrich, St. Louis, MO, USA) to remove L-[14C]-arginine. Columns were eluted two times with $0.5 \mathrm{ml}$ of distilled water and L-[14C]-citrulline was quantified using a Quantulus Liquid Scintillation Spectrometer (PerkinElmer, Inc., Waltham, MA, USA). cNOS activity was calculated by subtracting iNOS activity from total NOS activity. One unit (U) of total NOS activity was defined as picomoles of L-[14C]-citrulline produced per minute per microgram protein/ milliliter. The activity of cNOS in cells was expressed as U/mg of cell protein.

Western blotting for nNOS and c-jun expression. The expression of nNOS and c-jun in PC12 cells was determined by western immunoblot analysis performed as previously described $(19,20)$. In brief, differentiated PC12 cells following different treatments were harvested and lysed at $4^{\circ} \mathrm{C}$ by adding $100 \mu \mathrm{l}$ lysis buffer and $10 \mu \mathrm{l}$ phenylmethylsulfonyl fluoride $(100 \mathrm{~mm} / \mathrm{l})$ per $2 \times 10^{6}$ cells for $30 \mathrm{~min}$ on ice followed by centrifugation at $13,000 \times \mathrm{g}$ for $15 \mathrm{~min}$. Supernatant was collected for the determination of protein concentrations using the BCA protein assay using Bio-Rad Protein Assay Reagent (Bio-Rad, Hercules, CA, USA). The samples were stored at $-80^{\circ} \mathrm{C}$ until analysis (usually within 2 weeks). For western blotting, cell lysates (30 $\mu \mathrm{g}$ protein/lane) were separated on $8 \%$ sodium dodecyl sulphate-polyacrylamide gels with a pre-stained protein ladder $(5 \mu \mathrm{l})$ marker and electrophoretically transferred onto polyvinylidene difluoride membranes (Pall Corporation, Port Washington, NY, USA). The membranes were then blocked with $5 \%$ non-fat dry milk in tris-buffered saline (TBS) containing $0.1 \%$ Tween-20 at RT for $2 \mathrm{~h}$ and incubated with either c-jun polyclonal anti-rabbit antibody (1:1,000; Cell Signaling Technology), nNOS polyclonal anti-rabbit antibody (1:500; Cell Signaling Technology) or anti- $\beta$-actin monoclonal anti-mouse antibody $(1: 1,000$; Wuhan Boster Biological Technology, Ltd., Wuhan, China) overnight with gentle agitation at $4^{\circ} \mathrm{C}$. Following three washes in $0.1 \%$ Tween-20 in TBS (TBS-T) and incubation for $2 \mathrm{~h}$ at RT with horseradish peroxidase-conjugated polyclonal goat anti-rabbit secondary antibody (1:3,000; Cell Signaling Technology), the membranes were washed five times in TBS-T and immunocomplexes were visualized using the supersignal west Pico Trial kit (Pierce Biotechnology, Inc., Rockford, IL, USA) and the blots were exposed to X-ray film (Fujifilm, Tokyo, Japan). The intensity was quantified in the two bands. All bands on the immunoblots were normalized to their corresponding $\beta$-actin bands. Relative levels of protein in the different lanes were compared by analyzing scanned images using the NIH Image J software (National Institutes of Health, Bethesda, MA, USA). All experiments were performed three times with independent cultures.

Statistical analysis. All variables are presented as the mean \pm standard deviation of at least three independent experiments. Data analysis was performed using SPSS version 16.0 (SPSS, Inc., Chicago, IL, USA). One-way analysis of variance was used to analyze the differences among groups followed by Tukey-Kramer multiple comparison tests. $\mathrm{P}<0.05$ was considered to indicate a statistically significant difference.

\section{Results}

Expression of $c$-jun and $n N O S$ in differentiated PC12 cells in vitro. Differentiated PC12 cells were cultured as described in the experimental procedures. At first, the expression of nNOS and c-jun in differentiated PC12 cells was investigated by immunofluorescent double labeling detection. Morphologically, a large soma and two unbranched processes with the length of the processes being more than twice the length of the cell body, are indications of differentiation of PC12 cells. Under the DMI4000 B inverted microscope, it was observed that the bodies of incubated PC12 cells were round and transparent, and they were suspended in the cell culture solution evenly. The majority of the cells began to adhere to the wall of the cell culture dish at $4 \mathrm{~h}$ and stretched processes appeared $12 \mathrm{~h}$ after incubation, which were similar to the axons of neurons and their length was 2-fold longer than the diameter of cell bodies (Fig. 1). The results demonstrated that nNOS was expressed in differentiated PC12 cells, while nNOS immunoreactivity (ir) was present in the cytoplasm (Fig. 1A); c-jun was also clearly expressed in differentiated PC12 cells, while c-jun ir was present in the nuclei (Fig. 1D). All the nuclei of the differentiated PC12 cells were labeled with Hoechst 33258 (Fig. 1B and E). Immunofluorescence double labeling further indicated that c-jun and nNOS were co-expressed in almost all the differentiated PC12 cells (Fig. 1C and F). Based on the above findings, the next series of experiments aimed to determine the correlation between c-jun and nNOS in differentiated PC12 cells.

nNOS inhibitor 7-NI decreases the viability and survival of cultured differentiated PC12 cells in vitro. In order to examine the effectiveness of different concentrations of 7-NI and verify whether DMSO as the solvent of 7-NI had cell cytotoxicity, the PC12 cells were divided into the normal PC12 cell control group (normal group), DMSO control group (DMSO group) and 7-NI intervention group (7-NI group). The 7-NI group was subdivided into five different concentration groups of 10, 50, 100, 200 and $400 \mu \mathrm{mol} / 1$. With the 7-NI intervention for $24 \mathrm{~h}$, the cell viability was detected by MTT analysis. Statistical analysis demonstrated that the cell viability was significantly lower in the 200 and $400 \mu \mathrm{mol} / 1$ 7-NI treatment group compared with the normal group $(\mathrm{P}<0.05)$, while no significant difference was observed between the DMSO group and the normal group $(\mathrm{P}>0.05)$, which indicated that the DMSO had no cytotoxicity on differentiated PC12 cells (Fig. 2). Therefore, 

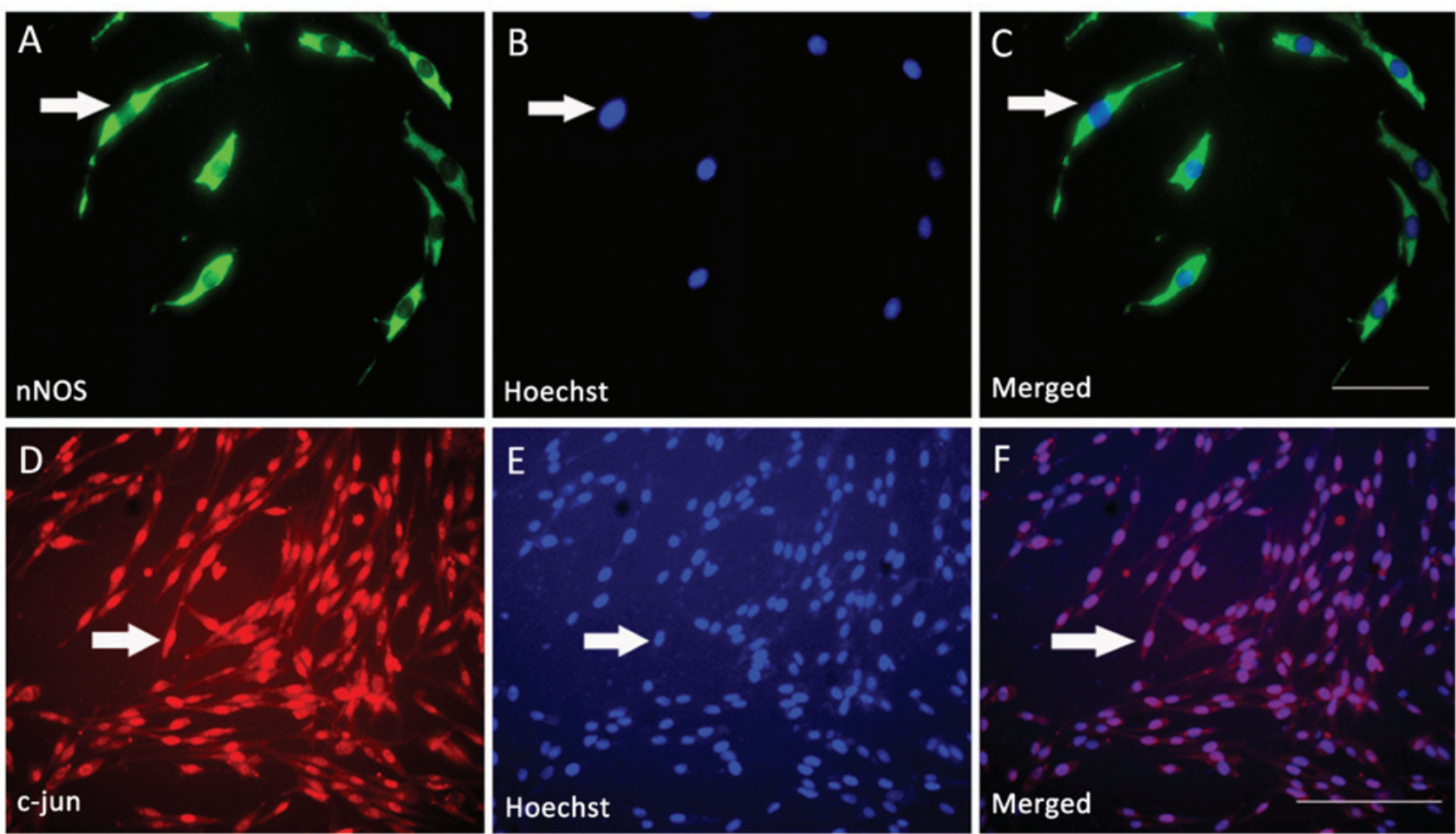

Figure 1. Expression of c-jun and nNOS by immunofluorescent double labeling in differentiated PC12 cells. (A) nNOS ir was present in the cytoplasm (green). (D) c-Jun ir was present in the nuclei (red). (B and E) All the nuclei of the differentiated PC12 cells were labeled with Hoechst 33258 (blue). ( $\mathrm{C}$ and $\mathrm{F}$ ) The results demonstrated that c-jun and nNOS were co-expressed in almost all the differentiated PC12 cells. (A-C magnification, x20; D-F magnification, x10). nNOS, neuronal nitric oxide synthase; ir, immunoreactivity.

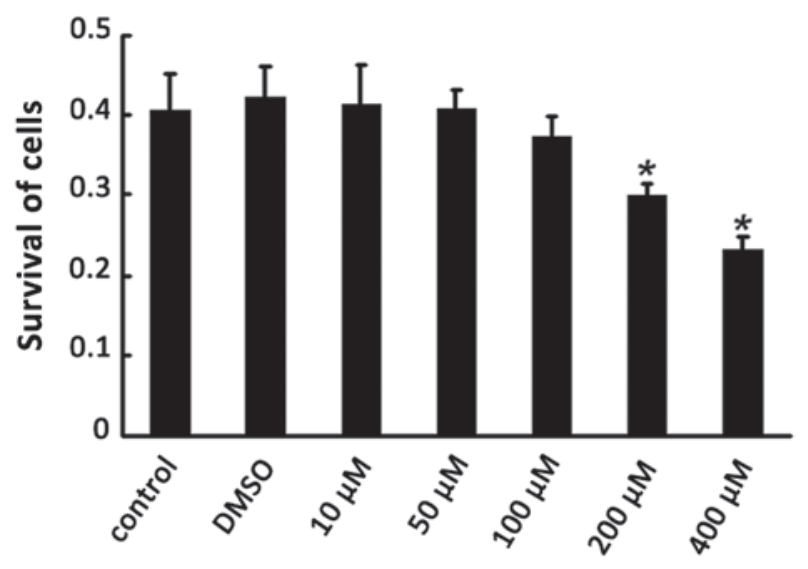

Figure 2. Effects of different concentrations of 7-NI on the cell viability of differentiated PC12 cells using a 3-(4,5-dimethylthiazol-2-yl)-2,5-diphenyltetrazolium assay. Statistical analysis demonstrated that 200 and $400 \mu \mathrm{mol} / 1$ 7-NI was able to markedly decrease the cell activities of differentiated PC12 cells compared with the normal group $\left({ }^{*} \mathrm{P}<0.05\right)$. DMSO, dimethyl sulfoxide; 7-NI, 7-nitroindazole.

$200 \mu \mathrm{mol} / 1$ 7-NI was selected as the optimal concentration for the following experiments.

nNOS inhibitor 7-NI downregulates the nNOS gene in differentiated PC12 cells. To investigate whether the nNOS inhibitor 7-NI reduces nNOS expression in differentiated $\mathrm{PC1} 2$ cells, the protein levels of nNOS in the cells following treatment with $200 \mu \mathrm{mol} / 1 \mathrm{7}-\mathrm{NI}$ for $24 \mathrm{~h}$ were evaluated by western blotting. The results indicated that the expression level of nNOS in the
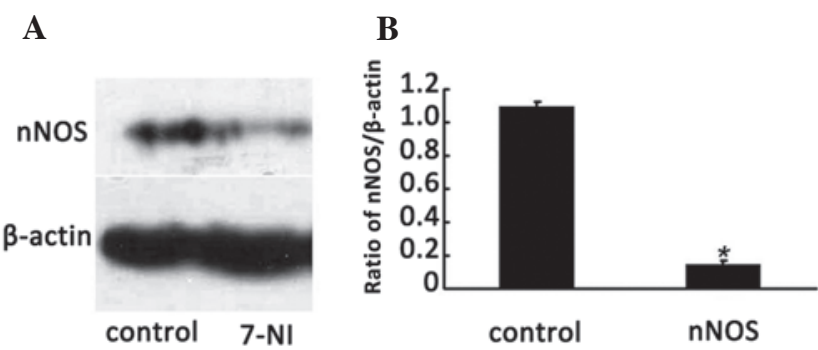

Figure 3. Effects of the nNOS inhibitor 7-NI on the nNOS gene in cultured differentiated PC12 cells. The results demonstrated that the nNOS protein was downregulated following treatment with 7-NI compared with the normal group $\left({ }^{*} \mathrm{P}<0.05\right)$ in differentiated $\mathrm{PC} 12$ cells. nNOS, neuronal nitric oxide synthase; 7-NI, 7-nitroindazole.

cells of the $200 \mu \mathrm{mol} / 1 \mathrm{7}$-NI group for $24 \mathrm{~h}$ was significantly lower than that of the normal group $(\mathrm{P}<0.05$; Fig. $3 \mathrm{~A}$ and $\mathrm{B})$, which indicated that the expression of the nNOS protein was able to be effectively inhibited by $200 \mu \mathrm{mol} / 17$-NI.

Effect of the nNOS inhibitor 7-NI on the activity of cNOS in differentiated PC12 cells. The nNOS inhibitor 7-NI also may result in a change in cNOS activity in various cell and tissue samples. The activity of cNOS was detected $24 \mathrm{~h}$ after treatment with $200 \mu \mathrm{mol} / 1$ 7-NI in differentiated PC12 cells. The total protein was extracted from the cells of the normal group, DMSO group and $200 \mu \mathrm{mol} / 1$ 7-NI group, and then the activity of cNOS in differentiated $\mathrm{PC} 12$ cells of these three groups were detected using the NOS Activity Detection kit. No significant differences were observed in the activity of cNOS among the $200 \mu \mathrm{mol} / \mathrm{l}$ 7-NI group, DMSO group and the control group 


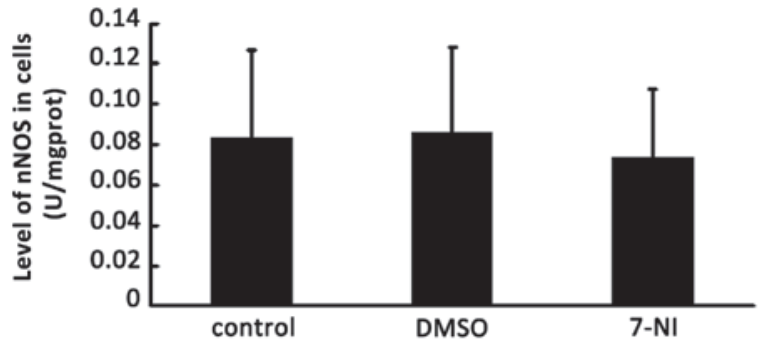

Figure 4. Effects of the nNOS inhibitor 7-NI on the activity of cNOS in differentiated PC12 cells using a NOS activity detection kit. No significant differences in the activity of cNOS between the 7-NI treatment group and the normal group were identified in differentiated PC12 cells $(\mathrm{P}>0.05)$. nNOS, neuronal nitric oxide synthase; cNOS, constitutive nitric oxide synthase; 7-NI, 7-nitroindazole; DMSO, dimethyl sulfoxide.

following intervention for $24 \mathrm{~h}$ (Fig. 4), which indicated that $200 \mu \mathrm{mol} / 17-\mathrm{NI}$ had no effect on the activity of cNOS in the differentiated PC12 cells.

Effect of the nNOS inhibitor 7-NI on the expression of $c$-jun in differentiated PC12 cells. Our previous study revealed that suppression of c-jun expression by c-jun siRNA decreased nNOS expression in differentiated PC12 cells (18). To clarify whether there was an association of up and downstream regulation or a crosstalk between c-jun and nNOS in neurons, the protein expression level of c-jun was detected by western blot analysis 24, 48 and $72 \mathrm{~h}$ after treatment with $200 \mu \mathrm{mol} / \mathrm{l}$ 7-NI. No significant differences were observed compared with the normal group and the DMSO group (Fig. 5), which indicated that the expression level of c-jun in differentiated PC12 cells was not affected by the inhibition of nNOS. The above results further verified that $\mathrm{nNOS}$, as a downstream signaling molecule of c-jun, regulated the survival of differentiated PC12 cells.

\section{Discussion}

In order to examine the association between c-jun and nNOS in neurons, the expression of c-jun and nNOS in differentiated PC12 cells was detected. In addition, the nNOS inhibitor 7-NI was used to downregulate the protein level of nNOS in order to observe the changes in the expression level of c-jun. The results demonstrated that $\mathrm{c}$-jun and $\mathrm{nNOS}$ were co-expressed in PC12 cells. Furthermore, the expression level of nNOS instead of the activity of nNOS was inhibited by $200 \mu \mathrm{mol} / 1$ 7-NI. In addition, the inhibited nNOS was not able to regulate the expression level of c-jun. Combined with our previous correlative research results, this indicated that the nNOS gene was a downstream signaling molecule of the JNK/c-jun signaling pathway in the model of differentiated PC12 cells. c-jun as an upstream signaling molecule was able to regulate the expression of nNOS downstream. Therefore, the association between them involved up and downregulation instead of crosstalk (1,16-19).

PC12 cells exhibit characteristic phenotypes of neurons following intervention with nerve growth factor (NGF), which is necessary for the differentiation of PC12 cells. In a study by Palmada et al (23), it was demonstrated that c-jun was required for sympathetic neuron degeneration following NGF
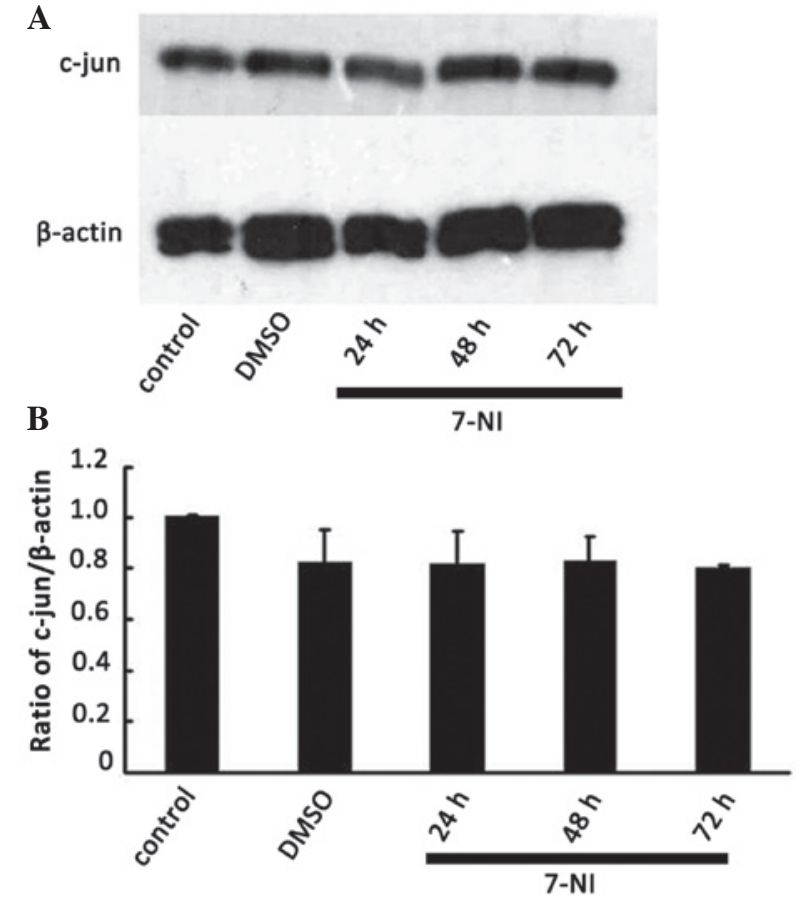

Figure 5. Effects of the nNOS inhibitor 7-NI on the c-jun gene in cultured differentiated PC12 cells. Cells were harvested 24, 48 and $72 \mathrm{~h}$ after treatment for western blot analysis of the c-jun protein. No significant differences were identified in the c-jun protein between the 7-NI treatment group and the normal group at different time points in differentiated $\mathrm{PC} 12$ cells $(\mathrm{P}>0.05)$. nNOS, neuronal nitric oxide synthase; 7-NI, 7-nitroindazole; DMSO, dimethyl sulfoxide.

withdrawal. It was reported that apoptosis of differentiated PC12 cells without NGF was inhibited by the complex dominant mutation of the c-jun gene and the injection of the specific antibody of c-jun (24). In addition, the constructed activity mutation of c-jun was a requirement for apoptosis caused by cerebellar granule cells (25). These findings indicated that the apoptosis observed in differentiated PC12 cells or other cells was mediated by the activation of c-jun whose mechanism remains to be elucidated. It was generally accepted that the above procedures were mainly due to the downstream target gene activated by c-jun in different types of cells or the protein that interacts with c-jun. Previous studies have demonstrated that the induced expression of c-jun and nNOS appeared in several animal models of neural diseases (15). For instance, nNOS and c-jun were co-expressed in the lateral geniculate nucleus in a rat model of experimental glaucoma (26) and the survival of hippocampal neurons was promoted by the $\mathrm{NO}$-induced activated JNK/c-jun in an animal model of ischemia (27). The impairment of proteasome activity and consequent increases in nNOS levels led to nitrative stress that involved the coordinated response of the JNK/c-jun cytosolic signaling pathway (28). All the above revealed that there was a certain functional association between c-jun and nNOS, which was consistent with our findings in differentiated PC12 cells in vitro (18). Previous studies have demonstrated that $\mathrm{c}$-jun and nNOS were co-expressed in motoneurons. In addition, the expression of nNOS was effectively downregulated by the activation of c-jun as an upstream signal (15). Furthermore, the expression of c-jun was present prior to that of nNOS due to the fact that the expression of nNOS was also downregulated by 
utilizing the c-jun siRNA to downregulate the expression level of c-jun in differentiated PC12 cells. However, the expression level of c-jun was not affected by utilizing 7-NI to downregulate the expression level of nNOS in the present study, which indicated that the nNOS gene was a downstream signaling molecule of the JNK/c-jun signal pathway. Additionally, c-jun as an upstream signaling molecule was able to regulate the expression of nNOS downstream, thus the association between them involved up and downregulation instead of crosstalk. The present study verified that nNOS was able to be regulated by c-jun as an upstream molecule, which expanded the theory of interaction between apoptosis and regenerated molecules in neurons. Although, this viewpoint requires further investigation by utilizing nNOS siRNA and the transgenic animal model of c-jun and nNOS.

In the present study, the expression level of nNOS was inhibited by an effective, cell-permeable, reversible nNOS inhibitor-7-NI. The results demonstrating that the activity of cNOS was not affected by 7-NI were mainly due to the following explanations: Firstly, the protein expression instead of the activity of nNOS protein was able to be regulated by 7-NI. In addition, cNOS could be subdivided into eNOS and nNOS, therefore the activity of cNOS was not equal to nNOS.

The present study revealed that c-jun and nNOS were co-expressed in differentiated PC12 cells, the expression level of nNOS protein was effectively inhibited by 7-NI and the expression level of c-jun was not affected by inhibition of nNOS. This indicated that the nNOS gene was a downstream signaling molecule of the JNK/c-jun signaling pathway, and that c-jun, as an upstream signaling molecule, was able to regulate the expression of nNOS downstream, thus the association between them involved up and downregulation instead of crosstalk. The current study clarified c-jun and nNOS as mediators of neuronal apoptosis, providing a greater understanding and enabling the application of molecular target therapy in neurodegenerative diseases.

\section{Acknowledgements}

This study was supported by research grants from the Natural Science Foundation Council of China (no. 81303115) and the Natural Science Foundation Council of Guangdong province (no. S2013040016915).

\section{References}

1. Wu W, Li L, Yick LW, et al: GDNF and BDNF alter the expression of neuronal NOS, c-Jun, and p75 and prevent motoneuron death following spinal root avulsion in adult rats. J Neurotrauma 20: 603-612,2003.

2. Ozdemir M, Attar A and Kuzu L: Regenerative treatment in spinal cord injury. Curr Stem Cell Res Ther 7: 364-369, 2012.

3. Förstermann U and Sessa WC: Nitric oxide synthases: regulation and function. Eur Heart J 33: 829-837, 2012.

4. Brown GC: Nitric oxide and neuronal death. Nitric Oxide 23: $153-165,2010$

5. Umar S and van der Laarse A: Nitric oxide and nitric oxide synthase isoforms in the normal, hypertrophic, and failing heart Mol Cell Biochem 333: 191-201, 2010.

6. Schonhoff CM and Bulseco DA: The Ras-ERK pathway is required for the induction of neuronal nitric oxide synthase in differentiating PC12 cells. J Neurochem 78: 631-639, 2001.
7. Raivich G: Transcribing the path to neurological recovery - from early signals through transcription factors to downstream effectors of successful regeneration. Ann Anat 193: 248-258, 2011.

8. Kenney AM and Kocsis JD: Peripheral axotomy induces long-term c-Jun amino-terminal kinase-1 activation and activator protein-1 binding activity by c-Jun and junD in adult rat dorsal root ganglia in vivo. J Neurosci 18: 1318-1328, 1998.

9. Mruthyunjaya S, Rumma M, Ravibhushan G, et al: c-Jun/AP-1 transcription factor regulates laminin-1-induced neurite outgrowth in human bone marrow mesenchymal stem cells: role of multiple signaling pathways. FEBS Lett 585: 1915-1922, 2011.

10. Ferrer I, Barrón S, Rodríquez-Farré E, et al: Ionizing radiation-induced apoptosis is associated with c-Jun expression and c-Jun/AP-1 activation in the developing cerebellum of the rat. Neurosci Lett 202: 105-108, 1995.

11. Fonseca MB, Nunes AF and Rodrigues CM: c-Jun regulates the stability of anti-apoptotic deltaNp63 in amyloid-beta-induced apoptosis. J Alzheimers Dis 28: 685-694, 2012.

12. Maritz MF, van der Watt PJ, Holderness N, et al: Inhibition of AP-1 suppresses cervical cancer cell proliferation and is associated with p21 expression. Biol Chem 392: 439-448, 2011.

13. Raivich G: c-Jun expression, activation and function in neural cell death, inflammation and repair. J Neurochem 107: 898-906, 2008.

14. Dragunow M, Xu R, Walton M, et al: c-Jun promotes neurite outgrowth and survival in PC12 cells. Brain Res Mol Brain Res 83: 20-33, 2000.

15. Wang LL, Zhao XC, Yan LF, et al: C-jun phosphorylation contributes to down regulation of neuronal nitric oxide synthase protein and motoneurons death in injured spinal cords following root-avulsion of the brachial plexus. Neuroscience 189: 397-407, 2011.

16. Zhou LH, Han S, Xie YY, et al: Differences in c-jun and nNOS expression levels in motoneurons following different kinds of axonal injury in adult rats. Brain Cell Biol 36: 213-227, 2008.

17. Zhou $\mathrm{L}$ and $\mathrm{Wu} \mathrm{W}$ : Antisense oligos to neuronal nitric oxide synthase aggravate motoneuron death induced by spinal root avulsion in adult rat. Exp Neurol 197: 84-92, 2006.

18. Cheng X, Liu S, Wang YQ, et al: Suppression of c-jun influences nNOS expression in differentiated PC12 cells. Mol Med Rep 6: 750-754, 2012.

19. Cheng X, Fu R, Gao M, et al: Intrathecal application of short interfering RNA knocks down c-jun expression and augments spinal motoneuron death after root avulsion in adult rats. Neuroscience 241: 268-279, 2013.

20. Li M, Wang L, Peng Y, et al: Knockdown of the neuronal nitric oxide synthase gene retard the development of the cerebellar granule neurons in vitro. Dev Dyn 239: 474-481, 2010.

21. Cheng X, Liu FL, Zhang J, et al: EGb761 protects motoneurons against avulsion-induced oxidative stress in rats. J Brachial Plex Peripher Nerve Inj 5: 12, 2010.

22. Li R, Wang WQ,Zhang H, et al: Adiponectin improves endothelial function in hyperlipidemic rats by reducing oxidative/nitrative stress and differential regulation of eNOS/iNOS activity. Am J Physiol Endocrinol Metab 293: 1703-1708, 2007.

23. Palmada M, Kanwal S, Rutkoski NJ, et al: c-jun is essential for sympathetic neuronal death induced by NGF withdrawal but not by p75 activation. J Cell Biol 158: 453-461, 2002.

24. Ham J, Babij C, Whitfeld J, et al: A c-Jun dominant negative mutant protects sympathetic neurons against programmed cell death. Neuron 14: 927-939, 1995.

25. Watson A, Eilers A, Lallemand D, et al: Phosphorylation of c-Jun is necessary for apoptosis induced by survival signal withdrawal in cerebellar granule neurons. J Neurosci 18: 751-762, 1998.

26. Wang X, Tay SS and Ng YK: C-fos and c-jun expressions in nitric oxide synthase immunoreactive neurons in the lateral geniculate nucleus of experimental glaucomatous rats. Exp Brain Res 144: 365-372, 2002.

27. Zeng XW, Li MW, Pan J, et al: Activation of c-Jun N-terminal kinase $1 / 2$ regulated by nitric oxide is associated with neuronal survival in hippocampal neurons in a rat model of ischemia. Chin Med J (Engl) 124: 3367-3372, 2011.

28. Lam PY and Cadenas E: Compromised proteasome degradation elevates neuronal nitric oxide synthase levels and induced apoptotic cell death. Arch Biochem Biophys 478: 181-186, 2008. 